\title{
The amended standard C37.118.1a and its implications for frequency-tracking M-class Phasor Measurement Units (PMUs)
}

\author{
Andrew J. Roscoe \\ Department of Electronic and Electrical Engineering \\ University of Strathclyde \\ Glasgow, UK \\ Andrew.J.Roscoe@strath.ac.uk
}

\author{
Bill Dickerson \\ Arbiter Systems \\ Paso Robles, CA, USA \\ Kenneth E. Martin \\ Electric Power Group \\ Portland, OR, USA
}

\begin{abstract}
The new amendment to the Phasor Measurement Unit (PMU) standard C37.118.1a makes several significant changes, compared to the standard C37.118.1 (2011). This paper highlights some of the most important changes, with a particular emphasis applied to how those changes relate to the way that a frequency-tracking PMU (Phasor Measurement Unit) algorithm needs to be designed. In particular, there is a delicate trade-off between passband flatness (the bandwidth test) and stopband rejection in the Out-Of-Band (OOB) test. For a PMU algorithm using frequency-tracking and adaptive filters, it is shown that passband flatness can be relaxed to about $2.5 \mathrm{~dB}$, but that the stopband needs to begin up to $14.8 \%$ closer to $0 \mathrm{~Hz}$ than for a fixed-filter PMU. This is partly due to the exact procedures of the C37.118.1a "OOB" testing, and partly due to the adaptive nature of a frequency-tracking PMU filter section.
\end{abstract}

Keywords-Power system measurements, Fourier transforms, Frequency measurement, Power system faults, Phase estimation, Power system state estimation, Power system parameter estimation

\section{TABLE OF NOMENCLATURE}

$\begin{array}{cl}A & \text { Amplitude of interharmonic }(\mathrm{pu}) \\ F() & \text { Baseband filter response } \\ F_{r} & \text { Bandwidth required } \\ F_{S} & \text { Reporting Rate }(\mathrm{Hz}) \\ f & \text { Fundamental frequency }(\mathrm{Hz}) \\ f_{0} & \text { Nominal frequency }(\mathrm{Hz}) \\ f_{I H} & \text { Interharmonic frequency }(\mathrm{Hz}) \\ f_{M} & \text { Modulation frequency }(\mathrm{Hz}) \\ f_{T} & \text { Tuned frequency for a tracking PMU }(\mathrm{Hz}) \\ H & \text { Per-unit inertia }(\mathrm{s}) \\ M & \text { Modulation depth }(\mathrm{pu})\end{array}$

\section{INTRODUCTION}

The present standard IEEE C37.118.1 was issued in 2011 [1] and presented a significant change relative to the 2005 standard. Specifically, dynamic requirements such as bandwidth, response time, delay time, latency, and frequency ramps were added. These are tested by applying amplitude and phase modulated signals, amplitude and phase steps, and frequency ramps. However, over the past 2 years some issues have been identified with the C37.118.1 (2011) standard. These are addressed with a new amendment C37.118.1a. In section III, some observations concerning requirements/limits in C37.118.1a are made, from an application standpoint.

However, the main focus of this paper is on the updated requirements for the bandwidth and Out-Of-Band interference (OOB) tests, and the implications for M-class Phasor Measurement Unit (PMU) design. The exact way these tests are carried out, and their stated limits, defines the way that filtering must be designed and implemented. In particular, it is shown in this paper that required filter masks are different for fixed-filter and frequency-tracking devices. In both implementations a quadrature oscillator is used to decode the phasor components from the AC signal using a heterodyne process. The fixed filter design keeps the quadrature oscillator at the nominal system frequency, $f_{0}$ where the frequency tracking filter adjusts the quadrature oscillator to the actual system frequency $f$. While a viable mask for fixed-filter devices is given in [2], a frequency-tracking PMU with adaptive filtering requires a different filter mask to comply with the standard. Equations developed in this paper allow such a mask to be created.

\section{THE REFERENCE ALGORITHM AND APPLICATION REQUIREMENTS}

Within C37.118.1, and C37.118.1a, a "Reference" PMU algorithm design is presented. It is important to note that the standard states "It is given for information purposes only, and does not imply being the only (or recommended) method for estimating synchrophasors. Its purpose is to establish common ground for understanding performance requirements and confirming their achievability". The reference model achieves

This is a postprint of a paper submitted to and presented at the IEEE International workshop on Applied Measurements for Power Systems (AMPS), 2014 [http://dx.doi.org/10.1109/AMPS.2014.6947702], and is subject to IEEE copyright. 
what it states above, but is not used in any commercial PMUs to our knowledge. Presently, C37.118.1a contains a series of tests with limits that can be complied with by the major PMU manufacturers, and also the Reference algorithm. This has required a certain degree of compromise in some areas. The tests and limits reflect performance achievable by both the Reference algorithm and all of the present major manufacturer's devices. The tests and limits do not necessarily reflect present of future application requirements, partly because these are not yet well quantified by the industry.

\section{CANDIDATE ALGORITHM DESIGNS}

A myriad of competing PMU algorithms have been proposed in literature and are appearing within actual PMUs. Some used fixed filters which remain centred on the nominal frequency. Such algorithms include the "C37.118.1a Reference", "Least Squares" [3] or "Taylor Fourier Transform" [4], and the "Interpolated DFT" [5] approaches. These approaches generally suffer from degraded performance as frequency deviates from nominal. Other algorithms use frequency-tracking, so that the algorithm tunes itself to constantly track the moving fundamental [6] [7] [8] [9]. This tends to decrease errors when frequency is off-nominal . If the filters are carefully designed, the filter notches (zeros) can also be moved to track harmonics, and even inter-harmonics if their frequency is known [9]. Most proposed algorithms use symmetric FIR windows since these most easily meet the C37.118.1a response and delay times. However, [9] proposes the use of Kalman filtering which is essentially an IIR technique. Many modern PMUs actually perform significantly better than the $\mathrm{C} 37.118 .1$ a limits.

\section{M-CLASS FILTER FLATNESS AND BANDWIDTH}

The bandwidth test is changed under C37.118.1a. Previously there were two tests: one with amplitude modulation only, and one with amplitude and phase modulation coincidentally. The combination of amplitude and phase modulation in C37.118.1 made it difficult to identify problems due to signal processing in this test, as the effects could sometimes occur destructively or constructively and in complex non-linear manners [10]. So, the amplitude and phase modulations are now applied separately in two sequential tests: with $0.1 \mathrm{pu}$ amplitude and then $0.1 \mathrm{rad}$ modulation applied. The required bandwidth of the PMU is set by a value $F_{r}$, calculated as $F_{r}=F_{S} / 5 \quad\left(F_{S}=\right.$ reporting rate $)$, but clipped to a maximum value of $5 \mathrm{~Hz}$.

The maximum allowable TVE (Total Vector Error) during this test is set at 3\% and it is interesting to analyse what this actually means. When the $0.1 \mathrm{pu}$ amplitude or $0.1 \mathrm{rad}$ phase modulation is applied to a $1 \mathrm{pu}$ fundamental at nominal frequency $f_{0}$, the true phasor should oscillate about the nominal $(1+0 j)$ point as shown in Fig. 1.

The length of trajectory for a $0.1 \mathrm{rad}$ disturbance is almost identical to the length for a $0.1 \mathrm{pu}$ amplitude disturbance since $\sin (\theta) \approx \theta$ when $\theta$ is small. A small phase modulation can therefore be analysed in the same way as an amplitude modulation.

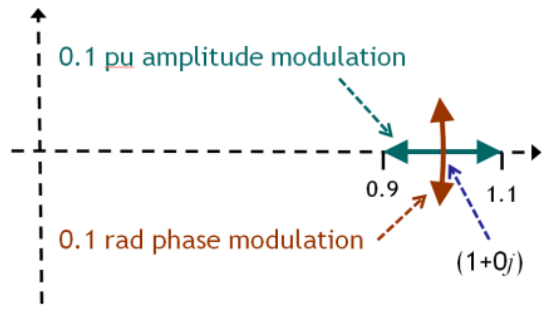

Fig. 1 Phasor oscillation during the bandwidth test

So, ignoring the "fixed" fundamental component at $(1+0 j)$ which is assumed to be accurately measured, the actual vector deviation expected in the presence of either amplitude or phase modulation can be evaluated by the following process:

The vector deviation from $(1+0 j)$ will have a form:

$V=\frac{M}{2} e^{j 2 \pi f_{M} t}+\frac{M}{2} e^{-j 2 \pi f_{M} t}$

where $M=0.1$ is the modulation depth, and $f_{M}$ is the modulation frequency, which can be a maximum of $F_{r}$. The two conjugate phasors represent phasors which add together to give the straight-line trajectories of Fig. 1.

When the waveform is measured, these deviations will be attenuated by the filter. The measured vector will appear as:

$V_{\text {Meas }}=F\left(f_{M}\right) \frac{M}{2} e^{j 2 \pi f_{M} t}+F\left(-f_{M}\right) \frac{M}{2} e^{-j 2 \pi f_{M} t}$

where $F\left(f_{M}\right)$ represents the baseband FIR filter response. The difference between these last two equations reveals the expected measurement error:

$$
\begin{aligned}
& T V E=\left|V_{\text {Meas }}-V\right| \\
& =\left|\left(F\left(f_{M}\right)-1\right) \frac{M}{2} e^{j 2 \pi f_{M} t}+\left(F\left(-f_{M}\right)-1\right) \frac{M}{2} e^{-j 2 \pi f_{M} t}\right|
\end{aligned}
$$

Now, for any FIR filter having only real weights:

$$
F\left(-f_{M}\right)=F^{*}\left(f_{M}\right)
$$

and also by setting $a=e^{j 2 \pi f_{M} t}$ as a unit phasor:

$$
T V E=\left|\left(F\left(f_{M}\right)-1\right) \frac{M}{2} a+\left(F *\left(f_{M}\right)-1\right) \frac{M}{2} a *\right|
$$

So:

$$
T V E=\left|\left(F\left(f_{M}\right)-1\right) \frac{M}{2} a+\left(\left(F\left(f_{M}\right)-1\right) \frac{M}{2} a\right) *\right|
$$

and it can be recognised that the sum of these conjugates equals the real part of their sum, and will form an oscillating TVE between zero and a maximum value.

$$
T V E=\left|M \times \operatorname{Re}\left(\left(F\left(f_{M}\right)-1\right) a\right)\right|
$$

Finally, assuming that the filter is symmetric about the timestamp $(t=0)$ in the time domain, the phase of the response

This is a postprint of a paper submitted to and presented at the IEEE International workshop on Applied Measurements for Power Systems (AMPS), 2014 [http://dx.doi.org/10.1109/AMPS.2014.6947702], and is subject to IEEE copyright. 
of $F\left(f_{M}\right)$ is zero for all $f_{M}$ and so $F\left(f_{M}\right)$ can be regarded as real, not complex. The maximum error at any instant in (7) will be:

$$
T V E_{\text {Max }}=M \times\left|\left(F\left(f_{M}\right)-1\right)\right|
$$

Therefore, the constraints on the gain $F\left(f_{M}\right)$ can be derived,

$\left|F\left(f_{M}\right)-1\right|<\frac{T V E_{\text {Limit }}}{M}$

Finally, re-ordering and substituting for $M=0.1$, $T V E_{\text {Limit }}=0.03(3 \%)$, this reveals:-

$\left|F\left(f_{M}\right)\right|>1-\frac{0.03}{0.1}$,i.e. $\left|F\left(f_{M}\right)\right|>0.7$

This means that the gain of the filter at the edge of the PMU bandwidth $\left(f_{M}=F_{r}\right)$ must be no lower than $-3.098 \mathrm{~dB}$. This $3 \mathrm{~dB}$ figure is not explicitly stated in the C37.118.1 text, but a recent NIST presentation alludes to this figure [10], as does [11], and the $3 \mathrm{~dB}$ point is a conventional way to describe bandwidth. Knowledge of this relationship is very useful for PMU design. For a given filter window length, a relaxed flatness requirement can allow an enhanced attenuation depth outside the passband, and this has clear benefits for broadband harmonic, inter-harmonic and noise rejection.

An asymmetric filter, such as a Kalman filter as proposed in [9], will have a non-zero phase $F\left(f_{M}\right)$ and so the gain at the band edge may need to be higher than $-3 \mathrm{~dB}$ for such a filter, to satisfy (9)-(10).

In other scenarios where amplitude and phase modulation occurs together, the vector deviation may be extremely complex due to non-linear interations caused by non-uniform filter window weights. If by some chance the deviation occurs in a in a perfect circular trajectory, then the analysis is simpler:

$$
\begin{aligned}
& V=M e^{j 2 \pi f_{M} t} \\
& V_{\text {Meas }}=F\left(f_{M}\right) M e^{j 2 \pi f_{M} t} \\
& T V E=\left|V_{\text {Meas }}-V\right|=\left|\left(F\left(f_{M}\right)-1\right) M e^{j 2 \pi f_{M} t}\right| \\
& T V E=\left|M \times\left(F\left(f_{M}\right)-1\right) a\right|
\end{aligned}
$$

In this case the TVE will be constant at the same maximum value as found in (7).

\section{A. Bandwidth in the Reference algorithm}

The Reference algorithm in C37.118.1a uses a flatness mask in the passband of $\pm 0.043 \mathrm{~dB}$ as part of its filter design process (Fig. 3). This value coincides with an error of $0.5 \%$ in absolute amplitude calibration. By using such a flat filter, as the fundamental frequency varies over the passband, no extra amplitude calibration needs to be calculated for the digital filter response, in order to meet a TVE of $1 \%$. This makes real-time computation easier within the Reference algorithm, but, as was shown above, a flatness of $\pm 0.043 \mathrm{~dB}$ is far tighter than is required to pass the bandwidth test, in terms of TVE.

\section{B. Bandwidth in a frequency-tracking or frequency- compensated algorithm.}

If the filter amplitude response is known and can be expressed mathematically using a fitted function or a lookup table, then the off-nominal filter gain can be accounted for, using the measured frequency value. This could be called a frequency-compensated PMU.

Alternatively, and additionally, if the quadrature oscillator [1] [7] tracks the measured fundamental frequency, so that the fundamental input frequency to the FIR filters is always moved towards $0 \mathrm{~Hz}$, then the deviation of the filter gain from unity is minimised, even during off-nominal frequency. This is a frequency-tracking PMU [7] [8] . Combining both approaches can lead to very low values of steady-state TVE as the fundamental frequency changes, but allows a relaxation of the flatness from $\pm 0.043 \mathrm{~dB}$ to nearly the $3 \mathrm{~dB}$ figure derived above.

In practice, it is found that setting a target filter gain of about $-2.5 \mathrm{~dB}$ leads to acceptable results in the bandwidth test, with highest TVE results in the region of $2.3-2.75 \%$. This margin gives a very low risk of unexpected compliance failures due to ADC resolution or noise since these tests are done with 1pu signals and all the modulated signal is in the passband.

\section{Frequency and ROCOF during bandwidth tests}

The original FE (Frequency Error) and RFE (Rate of change of Frequency Error) limits during bandwidth testing, for C37.118.1 (2011) were as shown in TABLE I. These presented a highly "quantised" FE and RFE performance which jumped suddenly at the $F_{S}=20 \mathrm{~Hz}$ boundary.

TABLE I. Bandwidth error limits - C37.118.1 (2011) [1]

\begin{tabular}{|c|c|c|c|c|}
\hline \multirow{2}{*}{$\begin{array}{c}\text { Modulation level, reference } \\
\text { condition, range } \\
\text { (use the same modulation levels } \\
\text { and ranges under the reference } \\
\text { conditions specified in Table 5) }\end{array}$} & \multicolumn{3}{|c|}{ Error requirements for compliance } \\
\cline { 2 - 5 } & Max FE & Max RFE & Max FE & Max RFE \\
\hline$F_{\mathrm{s}}>20$ & $0.06 \mathrm{~Hz}$ & $3 \mathrm{~Hz} / \mathrm{s}$ & $0.3 \mathrm{~Hz}$ & $30 \mathrm{~Hz} / \mathrm{s}$ \\
\hline$F_{\mathrm{s}} \leq 20$ & $0.01 \mathrm{~Hz}$ & $0.2 \mathrm{~Hz} / \mathrm{s}$ & $0.06 \mathrm{~Hz}$ & $2 \mathrm{~Hz} / \mathrm{s}$ \\
\hline
\end{tabular}

The problem with that "quantisation" was that an M-class PMU with a reporting rate $\left(F_{S}\right)$ of $15-20 \mathrm{~Hz}$ has quite a long window to meet $\mathrm{OOB}$ requirements, with high attenuation at the stop-band which begins at $F_{S} / 2$. But the bandwidth of such low-reporting-rate PMU's rises proportionately with $F_{r}=F_{S} / 5$ (Fig. 2). By contrast, PMUs with $F_{S} \geq 25$ have their bandwidth capped at $F_{r}=5 \mathrm{~Hz}$ (Fig. 2). So, as reporting rate rises from $F_{S}=10 \mathrm{~Hz}$ towards $F_{S}=25$, initially FE and RFE tend to increase as the bandwidth $F_{r}$ increases. But, as $F_{S}$ rises beyond $25 \mathrm{~Hz}$, the dominant effect is for a decrease in $\mathrm{FE}$ and a steady-state or slightly decreasing RFE, because $F_{r}$ is capped at $5 \mathrm{~Hz}$ and the filter gain can be increased towards $0 \mathrm{~dB}$ at $F_{r}$. Fig. 2 shows the required bandwidth $F_{r}$ (left-hand $\mathrm{Y}$ axis) and example $\mathrm{FE}$ results (right-hand $\mathrm{Y}$ axis) from a C37.118.1a-compliant frequency-tracking algorithm, for all valid $F_{S}$ suitable for 50 or $60 \mathrm{~Hz}$ systems. This highlights the problem with the C37.118.1-2011 limits, for $F_{S}=10$ to $F_{S}=20 \mathrm{~Hz}$ PMUs.

This issue has been resolved in C37.118.1a, by a new table (TABLE II) which allows FE and RFE to rise proportionately for low values of $F_{S}$, as bandwidth rises, but then FE and RFE are capped at upper values. By extending the mathematical analysis of (1)-(10) it would be possible to mathematically 
justify the new values, but Fig. 2 shows that the new C37.118.1a FE values are certainly achievable with filter gain at the band edge set close to the limit at $-2.5 \mathrm{~dB}$. Example results from testing both a frequency-tracking and fixed-filter (Reference) PMU are shown. Corresponding RFE results tell a similar story.

TABLE II. Bandwidth error limits - C37.118.1a [2]

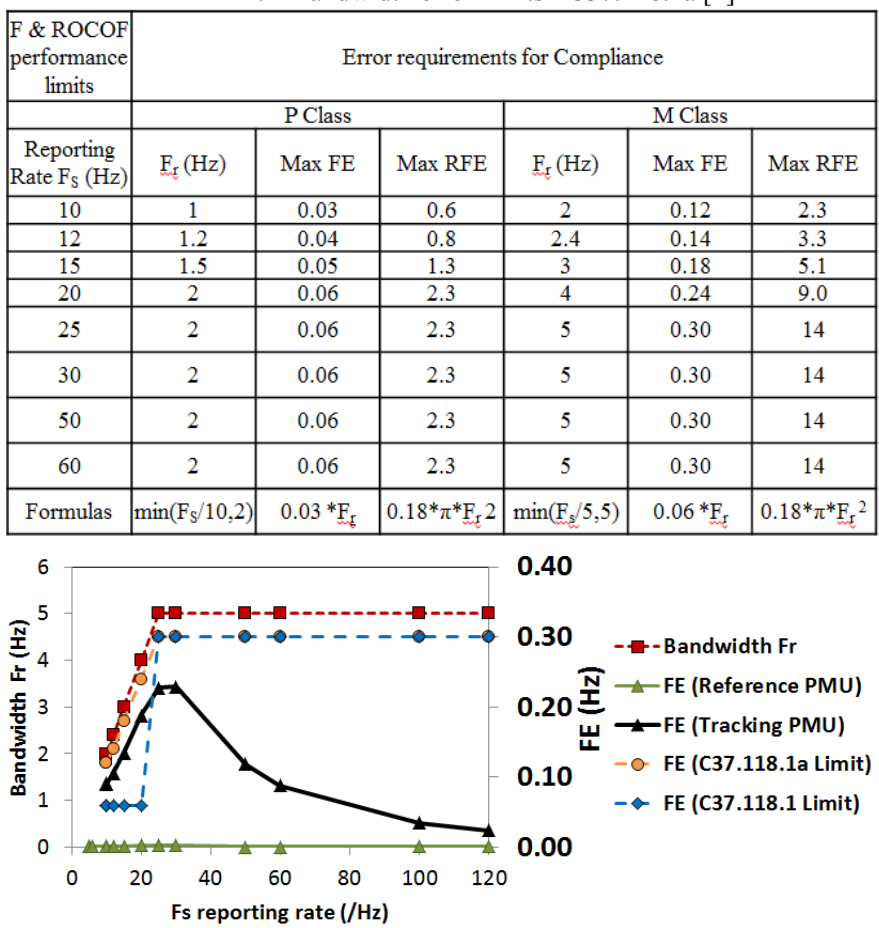

Fig. 2. Frequency Error (FE) example under bandwidth tests

\section{OUT OF BAND (OOB); STOP-BAND REJECTION}

The other major change in C37.118.1a is within the OOB test, and requires great care in the filter design. In the original C37.118.1, the emphasis was on achieving a $1.3 \%$ TVE result when the $10 \%$ OOB interharmonics were applied. This corresponded to a $20 \mathrm{~dB}$ stop-band rejection, which defined the original Reference algorithm mask. However, C37.118.1 also contained OOB FE and RFE limits which could not met by its Reference algorithm, with just 20dB stopband rejection.

In C37.118.1a, the OOB RFE limit has been suspended. But, the OOB FE limit of $0.01 \mathrm{~Hz}$ has been retained. This is significant because it requires $>20 \mathrm{~dB}$ of stopband rejection. It is now the OOB FE limit, not the 1.3\% TVE limit, which defines the filter mask. The new mask for the Reference algorithm is shown in Fig. 3. To achieve the increased stopband rejection, using the Reference algorithm, requires longer time windows and slightly adjusted filters compared to those given in C37.118.1. This has, in turn, required longer (relaxed) response and latency limits to be applied in C37.118.1a. Many PMUs may exhibit significantly shorter responses and latencies than the $\mathrm{C} 37.118$.1 a limits.

\section{A. Determining the required $O O B$ filter rejection}

It is possible to calculate the required baseband filter rejection $F($ ), which is a function of frequency separation from the tuned frequency $f_{T}$. (For a fixed-filter PMU $f_{T}=f_{0}$, and for a tracking $\operatorname{PMU} f_{T} \approx f$ ).

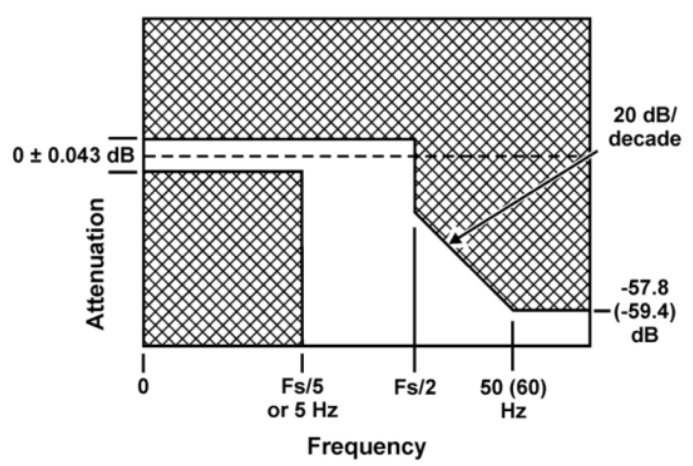

Fig. 3 Filter mask for the C37.118.1a Reference algorithm [2]

If an interharmonic of amplitude $A$ applied at frequency $f_{I H}$, while the fundamental signal has amplitude 1 pu at frequency $f$.

$V_{\text {in }}=e^{j 2 \pi f t}+A e^{j 2 \pi f_{I H} t}$

When the heterodyning with the quadrature oscillator and then filtering $F()$ are applied, with both tuned to expect an fundamental frequency $f_{T}$, the perceived measurement vector at the filter output will consist of the dominant fundamental with a magnitude 1pu, upon which is superimposed another trajectory, which will in the worst case will be circular, similarly to (11).

$$
\begin{aligned}
& V_{\text {Heterodyned }}=e^{-j 2 \pi f_{T} t}\left(e^{j 2 \pi f t}+A e^{j 2 \pi f_{I H} t}\right) \\
& V_{\text {Heterodyned }}=e^{j 2 \pi\left(f-f_{T}\right) t}+A e^{j 2 \pi\left(f_{I H}-f_{T}\right) t} \\
& V_{\text {Meas }}=F\left(f-f_{T}\right) e^{j 2 \pi\left(f-f_{T}\right) t} \\
& \quad+F\left(f_{I H}-f_{T}\right) A e^{j 2 \pi\left(f_{I H}-f_{T}\right) t} \\
& V_{\text {Meas }}=e^{j 2 \pi\left(f-f_{T}\right) t} \times \\
& {\left[F\left(f-f_{T}\right)+A F\left(f_{I H}-f_{T}\right) e^{j 2 \pi\left(f_{I H}-f\right) t}\right]}
\end{aligned}
$$

Assuming that filter gain $F\left(f-f_{T}\right)$ is almost unity, the measurement error is due to the second term. Frequency error can be evaluated by examining the worst-case effect of this on the phase of the (otherwise steady) phasor $\Delta V_{\text {Meas }}$ where:

$$
\begin{aligned}
& \Delta V_{\text {Meas }}=V_{\text {Meas }} e^{-j 2 \pi\left(f-f_{T}\right) t} \\
& \Delta V_{\text {Meas }}=\left(1+A F\left(f_{I H}-f_{T}\right) e^{j 2 \pi\left(f_{I H}-f\right) t}\right)
\end{aligned}
$$

It can be seen that $\Delta V_{\text {Meas }}$ consists of a small circular error trajectory centred on the point $(1+0 \mathrm{j})$. The effect on frequency measurement is determined by the maximum rate of change of phase error $\Delta \phi$ :

$$
\Delta \phi=\angle\left(\Delta V_{\text {Meas }}\right)
$$

The biggest rate of change of $\Delta \phi$ will occur when at the closest approach of $\Delta V_{\text {Meas }}$ to the origin $(0+0 j)$, when $2 \pi\left(f_{I H}-f\right) t=\pi$. At this instant $\Delta V_{\text {Meas }}$ has its lowest magnitude, with a value of $\left(\left(1-A F\left(f_{I H}-f\right)\right)+0 \mathrm{j}\right)$ but also has its maximum velocity parallel to the $j$ axis: $-2 \pi\left(f_{I H^{-}} f\right) A F\left(f_{I H^{-}} f_{T}\right) \mathrm{s}^{-1}$. Therefore,

This is a postprint of a paper submitted to and presented at the IEEE International workshop on Applied Measurements for Power Systems (AMPS), 2014 [http://dx.doi.org/10.1109/AMPS.2014.6947702], and is subject to IEEE copyright. 
the maximum magnitude of the rate of change of phase error $\Delta \phi$ is:

$$
\begin{aligned}
& \left|\frac{d(\Delta \phi)}{d t}\right|_{\text {Max }}=\left|\frac{-2 \pi\left(f_{I H}-f\right) \cdot A \cdot F\left(f_{I H}-f_{T}\right)}{\left(1-A \cdot F\left(f_{I H}-f_{T}\right)\right)}\right| \\
& F E_{\text {Max }}=\frac{1}{2 \pi} \cdot\left|\frac{d(\Delta \phi)}{d t}\right|_{\text {Max }}
\end{aligned}
$$

and therefore:

$$
F\left(f_{I H}-f_{T}\right)<\frac{F E_{\text {Max }}}{A\left(\left|f_{I H}-f\right|+F E_{\text {Max }}\right)}
$$

Since $F E_{\text {Max }}=0.01 \mathrm{~Hz}$, and the closest OOB frequency for the $F_{S}=10 \mathrm{~Hz}$ PMU is $5 \mathrm{~Hz}, F E_{M a x}<<\left|f_{I H-} f\right|$ and so the following approximation is valid:

$$
F\left(f_{I H}-f_{T}\right)<\frac{F E_{M a x}}{A \cdot\left|f_{I H}-f\right|}
$$

This defines the highest filter gain which can possibly satisfy the OOB test. In practice, to allow a safety margin for noise, Effective Number of Bits (ENOB) of ADC resolution etc., it is sensible to allow reduce this figure by $3 \mathrm{~dB}$.

$$
F\left(f_{I H}-f_{T}\right)<\left(\frac{F E_{M a x}}{A \cdot\left|f_{I H}-f\right|}\right) 10^{\left(\frac{-3}{20}\right)}
$$

However, the way that (27) needs to be evaluated is different for fixed-filter and frequency-tracking PMUs, as is the range of values of $f_{I H}$ which should in theory be applied to validate the performance.

\section{B. Fixed-filter algorithms}

For any interharmonic such as flicker at frequency $f_{I H}$, the ripple frequency of error at the PMU output, due to the heterodyning process, is given by:

$$
f_{\text {ripple }}=\left|f_{I H}-f_{T}\right|
$$

The aim of the C37.118.1a OOB test is to verify a filter stopband which limits any measurement ripples with frequencies $>=F_{S} / 2$. Therefore:

$$
\left|f_{I H}-f_{T}\right| \geq \frac{F_{S}}{2}
$$

For fixed-filter PMUs such as the Reference algorithm, $f_{T}=f_{0}$ always, and so:

$$
\text { Fixed - filter stopband : }\left|f_{I H}-f_{0}\right| \geq \frac{F_{S}}{2}
$$

This matches exactly with the C37.118.1a OOB test, where the stopband is defined exactly as (30). C37.118.1a testing applies (30) but only varies fundamental frequency over a reduced range compared to the whole valid range $\left(f_{0} \pm F_{r}\right)$ :

$$
f \text { range: }\left(f_{0}-\frac{F_{S}}{20}\right) \leq f \leq\left(f_{0}+\frac{F_{S}}{20}\right)
$$

For fixed-filter devices, on the left hand side (LHS) of (27), the mask always refers to $F\left(f_{I H}-f_{0}\right)$. But, on the right hand side (RHS), there is a worst case during C37.118.1a testing where $\left(f_{I H}-f\right)$ reaches $\left(F_{S} / 2+F_{S} / 20\right)=11 F_{S} / 20$, i.e. a $10 \%$ increase from $F_{S} / 2$. This $10 \%$ increase means that the gain $F()$ needs to be $20 * \log (1.1)=0.83 \mathrm{~dB}$ reduced from a value obtained via a more "naïve" analysis by (for example, and incorrectly):

$$
F\left(f_{I H}-f_{0}\right)<\left(\frac{F E_{M a x}}{A \cdot\left|f_{I H}-f_{0}\right|}\right) 10^{\left(\frac{-3}{20}\right)}
$$

Careful evaluation of (27), in the above configuration, leads to the mask given for the C37.118.1a Reference algorithm.

\section{Frequency-tracking algorithms}

The effect and analysis of interharmonics within a frequency-tracking device is different. In such a device, $f_{T}=f$ if the algorithm is tracking correctly. Therefore, to define the required stopband from (28) and (29):

$$
\text { Tracking stopband : }\left|f_{I H}-f\right| \geq \frac{F_{S}}{2}
$$

Equation (33) implies that a different regime from (30) should ideally be used to test a frequency-tracking device. The difference between (30) and (33) is small when $f \approx f_{0}$ but becomes significant as the fundamental frequency $f$ deviates from nominal.

The actual C37.118.1a test applies the regime in (30) and (31). The issue here is that the lowest ripple frequency appearing after the heterodyning during the test regime is given by (28) with (as a worst-case example) $f_{T}=f=\left(f_{0}+F_{S} / 20\right) \mathrm{Hz}$ and $f_{I H}=\left(f_{0}+F_{S} / 2\right) \mathrm{Hz}$. This requires a stopband width of only $9 F_{S} / 20 \mathrm{~Hz}$, which is $10 \%$ narrower than the stopband required using a fixed-filter device to pass the same test.

Therefore, it is harder for a frequency-tracking algorithm to pass the C37.118.1a OOB test than for a fixed-filter algorithm to. The filter has to be more aggressively designed to achieve stopband cutoff at a baseband filter frequency which is $10 \%$ lower than the equivalent fixed-filter design. Making use of the available $\sim 2.5 \mathrm{~dB}$ filter flatness relaxation is one way to try and achieve this more aggressive cutoff.

Notice that if the testing regime was changed to (33) for the frequency-tracking device, this $10 \%$ narrowing would no longer be required. Also, in that case, the full valid fundamental input frequency range $\left(f_{0} \pm F_{r}\right)$ (and potentially much wider) could be tested, using the same baseband filter design, and the same $0.01 \mathrm{~Hz}$ FE might still be attained, or nearly attained (but see section $\mathrm{D}$ below).

The required attenuation can again be evaluated by (27). For a frequency-tracking $\mathrm{PMU}, f_{T}=f$ if the algorithm is tracking correctly. This means there is no deviation between the frequencies on the LHS and RHS of (27) and the $0.83 \mathrm{~dB}$ factor is not required. In fact, the gain of the filter can be $0.92 \mathrm{~dB}$ higher at the lowest required frequency $\left(f_{I H}-f_{T}\right)$, than the simplistic analysis of (32) suggests, since $\left(f_{I H}-f\right)$ is also reduced by a factor 0.9 at the same critical point. This works slightly in favour of the tracking PMU during the OOB test, but

This is a postprint of a paper submitted to and presented at the IEEE International workshop on Applied Measurements for Power Systems (AMPS), 2014 [http://dx.doi.org/10.1109/AMPS.2014.6947702], and is subject to IEEE copyright. 
not enough to balance the difficulty introduced by the $10 \%$ stopband reduction.

\section{Accounting for adaptive filter shortening}

There is a further complication for tracking algorithms, which is that the entire filter window design may adapt linearly to become shorter as the measured fundamental frequency increases (to track the filter notches with the harmonics). Tracking algorithms such as [7] [8] fall into this category. This will expand the filter frequency response proportionately. Therefore, when designing such an adaptive filter for the baseline $f_{0}$ case, the mask window width in frequency may need to be further reduced by a proportional factor of:

$$
\text { Adaptive Mask Width Factor }=f_{0} /\left(f_{0}+\frac{F_{S}}{20}\right)
$$

Otherwise, a tracking PMU will likely fail an OOB test when $f=\left(f_{0}+F_{S} / 20\right)$. Examples of the factor in (34) are 0.99 (a $1 \%$ reduction) for $F_{S}=10 \mathrm{~Hz}$ and 0.952 (a $4.8 \%$ reduction) for $F_{S}=50 \mathrm{~Hz}$, with $f_{0}=50 \mathrm{~Hz}$. This reduction is not due to the C37.118.1 a test process regime, but is inherent in a frequencytracking device with such adaptive filters.

\section{OVERALL EFFECTS ON THE FILTER MASK FOR FREQUENCY-TRACKING ALGORITHMS}

The total effect is that the width of the stopband mask for a frequency-tracking algorithm is reduced by between $11 \%$ and $14.8 \%$ compared to the mask for a fixed filter, when the filter is designed at $f_{0}$ and the $\mathrm{C} 37.118 .1 \mathrm{a}$ OOB test procedure is to be applied. As an example, Fig. 4 shows the masks and filter envelopes for the C37.118.1a Reference algorithm, and a C37.118.1a-compliant tracking algorithm, in the $f=f_{0}=F_{S}=50 \mathrm{~Hz}$ case. The mask frequency-width reductions required for the tracking filter $(10 \%+4.8 \%)$ have been indicated, showing how the mask stopband begins almost $15 \%$ closer to the passband than $F_{S} / 2$. Both masks are calculated using (27) and the procedures of section VI B and VI C.

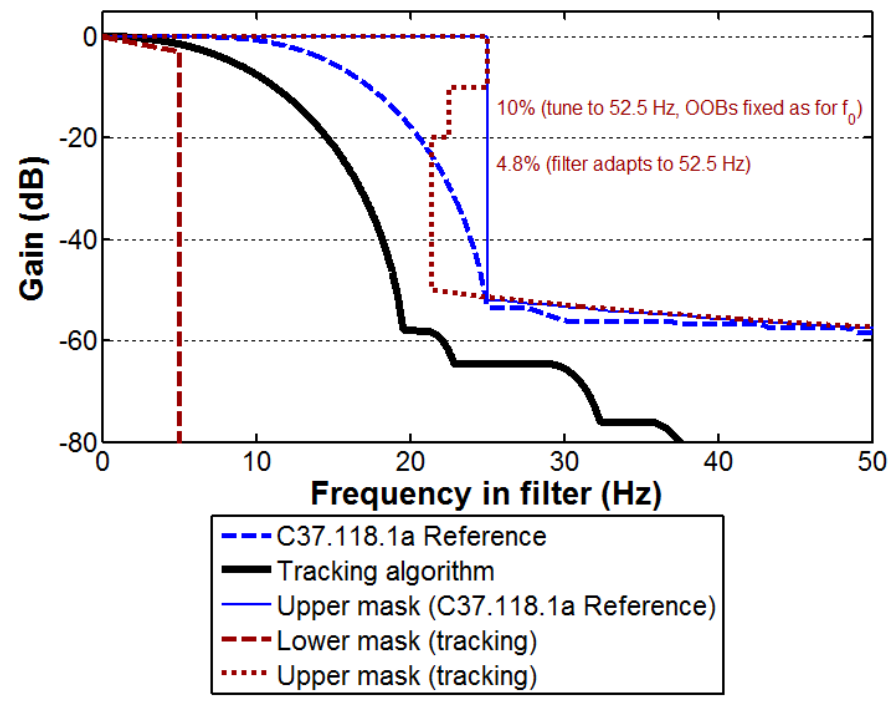

Fig. 4 Example of filter envelopes and masks for the Reference algorithm, and a C37.118.1 a-compliant tracking algorithm. $f=f_{0}=F_{S}=50 \mathrm{~Hz}$.

\section{CONCLUSIONS}

The new standard C37.118.1a-2014 is a useful amendment to C37.118.1-2011. The Reference algorithm within C37.118.1a is now compliant, as well as PMUs from most (if not all) major PMU manufacturers. There are some areas where, from an application perspective, limits (particularly RFE) would ideally be tightened or additional scenarios tested, but this really requires a greater engagement between the PMU user community and the C37.118.1a standard committee, to agree on realistic conditions, evidence, expectations and limits.

Implementing a good filter and algorithm requires a careful balance between, in particular, the bandwidth and OOB tests. These tests have both altered under C37.118.1a. Theoretical derivations in this paper, backed up with C37.118.1a tests in a simulated environment, show that the passband flatness can be relaxed to about $2.5 \mathrm{~dB}$ for a frequency-compensated or frequency-tracking PMU. In these PMUs, the reduced flatness can be traded off, in order to provide a faster cutoff to the stopband and a deeper broadband stopband rejection.

Filter masks for both fixed-filter and frequency-tracking devices have been derived. The mask defining the beginning of the stopband needs to be up to $14.8 \%$ narrower for a frequencytracking PMU than for a fixed-filter PMU. This is partly due to the exact way that the present OOB test is conducted, and partly due to the inherent frequency-tracking property.

\section{REFERENCES}

[1] IEEE, "IEEE Standard for Synchrophasor Measurements for Power Systems," C37.118.1-2011, 2011.

[2] IEEE, "IEEE Standard for Synchrophasor Measurements for Power Systems -- Amendment 1: Modification of Selected Performance Requirements " C37.118.1a-2014, 2014.

[3] D. Belega and D. Petri, "Performance of Synchrophasor Measurements Provided by the Weighted Least Squares Approach," in IEEE I2MTC 2013, Minneapolis, 2013, pp. 946-951.

[4] P. Castello, M. Lixia, C. Muscas, and P. A. Pegoraro, "Adaptive TaylorFourier synchrophasor estimation for fast response to changing conditions," 2012 IEEE International Instrumentation and Measurement Technology Conference (I2mtc), pp. 294-299, 2012.

[5] D. Belega and D. Petri, "Accuracy Analysis of the Multicycle Synchrophasor Estimator Provided by the Interpolated DFT Algorithm," IEEE Transactions on Instrumentation and Measurement, vol. 62, pp. 942-953, May 2013.

[6] J. A. D. Serna, "Synchrophasor Estimation using Prony's Method," IEEE Transactions on Instrumentation and Measurement, vol. 62, 2013.

[7] A. J. Roscoe, I. F. Abdulhadi, and G. M. Burt, "P and M Class Phasor Measurement Unit Algorithms using Adaptive Cascaded Filters," IEEE Transactions on Power Delivery, vol. 28, pp. 1447-1459, 2013.

[8] A. J. Roscoe, "Exploring the relative performance of frequency-tracking and fixed-filter Phasor Measurement Unit algorithms under C37.118 test procedures, the effects of interharmonics, and initial attempts at merging P class response with M class filtering," IEEE Transactions on Instrumentation and Measurement, vol. 62, pp. 2140-2153, 2013.

[9] I. Kamwa, S. R. Samantaray, and G. Joos, "Wide Frequency Range Adaptive Phasor and Frequency PMU Algorithms," IEEE Transactions on Smart Grid, vol. 5, 2014.

[10] National Institute of Standards and Technology (NIST), "Lessons learned from the NIST assessment of PMUs," 2014 Available: https://www.naspi.org/File.aspx?fileID=1250, accessed May 2014.

[11] K. E. Martin, B. Kasztenny, M. G. Adamiak, G. Antonova, M. Begovic, et al., "Synchrophasor Measurements under the IEEE Standard C37.118.1-2011 with amendment C37.118.1a," IEEE Transactions on Power Delivery, In Review. 